\title{
EDITORIAL Effective population size in ecology and evolution
}

\author{
Heredity (2016) 117, 191-192; doi:10.1038/hdy.2016.75; published online 24 August 2016
}

Effective population size $\left(N_{\mathrm{e}}\right)$ is one of the most important parameter in population genetics and conservation biology. It translates census sizes of a real population into the size of an idealized population showing the same rate of loss of genetic diversity as the real population under study. Several conceptually different types of $N_{\mathrm{e}}$ can be distinguished, but the most commonly used ones are those based on the loss of genetic diversity through inbreeding (inbreeding $N_{\mathrm{e}}$ ) and through genetic drift (variance $N_{\mathrm{e}}$ ). Often, these two differ considerably, as do census and effective sizes, although, contrary to what is taught in many introductory courses and textbooks, $N_{\mathrm{e}}$ is not always lower than census size (Braude and Templeton, 2009, see also Nunney in this issue). Inbreeding and variance $N_{\mathrm{e}}$ particularly differ when demography changes. However, these relationships are not all that well understood yet, and require further theoretical and empirical attention.

Many recent developments in theory, modeling and statistics as well as in molecular biology have sparked new interest in prediction and estimation of $N_{\mathrm{e}}$ and have led to a number of papers documenting recent advancements in the field. This special issue comprises a collection of papers that tackle various theoretical and applied aspects concerning the estimation of $N_{\mathrm{e}}$ under simulated conditions and in natural situations. Contributions were chosen to provide a balanced overview of the topic by summarizing the state of the art (Wang et al. in this issue), providing new developments in theory, improvements in estimation methods, especially in terms of applicability to genomewide data (Hollenbeck et al.; Jones et al.; Nunney; Waples et al.; Waples, all in this issue), and some case studies employing currently available methods (Coscia et al.; Cousseau et al.; Ferchaud et al.; Laikre et al.; Mueller et al., all in this issue). Although not being comprehensive, the special issue hopes to provide a good overview of the current status of the field as well as providing new advancements.

Wang et al. present a review across the discipline, focusing on the distinction of prediction and estimation methods of $N_{\mathrm{e}}$. The authors provide a general introduction into the field and describe the theoretical background of current prediction and estimation methods in depth, serving as an introduction to the topic.

The next five papers provide technical and theoretical advancements in estimation methods. The contributions of this part are focusing on new methods addressing specific problems in estimating $N_{\mathrm{e}}$ under natural conditions, several specifically on the linkage disequilibrium (LD) method. The LD method is currently the most used as it allows robust estimation of $N_{\mathrm{e}}$ from samples taken at only a single point in time. One of the big challenges is to adjust these methods for estimating $N_{\mathrm{e}}$ to genomic data, which are often available as Single Nucleotide Polymorphisms (SNPs). Estimating $N_{\mathrm{e}}$ from SNPs is the focus of Waples et al., who adopt the LD method to genomic data sets. The problem with such data is physical linkage of some of the loci, which may bias estimates downwardly. The authors used simulations to show that linkage does not overall increase with higher numbers of loci. Nevertheless, some loci inevitably show strong linkage. The authors present a method to reduce this bias by taking chromosome number and length into account. Yet, the precision of estimates based on SNPs is lower than expected. Jones et al. tackle the problem of reliability of confidence intervals (CIs). They provide two new methods to generate CIs and compare them with previous parametric and jackknife techniques. The new methods are specifically applicable when using large numbers of genomic SNPs and hence represent valuable alternatives for genome-wide data sets. Hollenbeck et al. also use the LD method, but employ it to test for recent changes in $\mathrm{N}_{e}$ using SNP markers. The authors test their new approach on an empirical data set of marine fish in which a recent decline is known to have occurred. They show that even small and recent changes can be detected and thus provide a valuable new tool for conservation studies. A more specific problem with estimating $N_{\mathrm{e}}$ is addressed by Nunney, whose paper clarifies the distinction of $N_{\mathrm{e}}$ and the effective size of a population's neighborhood $\left(N_{\mathrm{n}}\right)$ and also investigates the effects of $N_{\mathrm{n}}$ on $N_{\mathrm{e}}$ using simulations. $N_{\mathrm{n}}$ is primarily based on the dispersal characteristics of a species and is independent of $N_{\mathrm{e}}$. Simulations show that the effects of $N_{\mathrm{n}}$ on $N_{\mathrm{e}}$ can be substantial when sampling is temporal; the author provides an optimal sampling scheme to reduce such bias and suggests that low effective to census size ratios $\left(N_{\mathrm{e}} / N\right)$ may be a result of the bias. In addition to genetic methods, $N_{\mathrm{e}}$ can also be inferred from life history traits. The contribution by Waples reiterates the importance of considering life history traits, specifically those related to adult mortality, and discusses problems with estimating $N_{\mathrm{e}}$ when generations overlap. The author implements life history parameters related to adult mortality in predictive equations and tests these on an empirical data set of 63 animal and plant species. He suggests that a comparison of non-genetic and genetic estimates of $N_{\mathrm{e}}$ may provide valuable information to interpret such estimates in the future.

The third block of papers presents applied studies, where diverse methods of $N_{\mathrm{e}}$ estimation are employed in different contexts and under different natural settings. Ferchaud et al. investigate and compare $N_{\mathrm{e}}$, the number of breeders $\left(N_{\mathrm{b}}\right)$ and the census size $\left(N_{c}\right)$ in Atlantic salmon. They use temporal genetic data to develop models for predicting $N_{\mathrm{c}}$ from $N_{\mathrm{b}}$. The authors find a high variance in the predictive power of the model and conclud that thorough calibration is necessary before using $N_{\mathrm{b}}$ in conservation studies. Coscia et al. use a different fish system, two species of sea breams with different breeding systems, to investigate the influence of sex change on the estimation of $N_{\mathrm{e}}$. The authors find that gonochoristic species have lower contemporary $N_{\mathrm{e}}$. Yet, this difference was not found across historical time scales. The authors conclude that sex change may influence $N_{\mathrm{e}}$ differently over varying time frames, but that this variance may also depend on the genetic markers used to infer 
demographic parameters. Cousseau et al. use historic and contemporary samples to test for temporal shifts of genetic diversity and $N_{\mathrm{e}}$ in a recently collapsed house sparrow population in the Netherlands. They find no change in genetic diversity before and after the collapse, but detect an increase in differentiation, suggesting that levels of gene flow have declined. Further, $N_{\mathrm{e}}$ was found to be reduced. The study unravels genetic signatures supporting the decline of house sparrows in Europe and underlines the value of historical samples in conservation genetic studies (Habel et al., 2014). Mueller et al. use a large data set of a longterm monitored buzzard population to evaluate single-sample and temporal methods of estimating $N_{\mathrm{e}}$. The monitoring data further allow the authors to compare $N_{\mathrm{e}}$ and $N_{\mathrm{c}}$ estimates across multiple years. Temporal and single-sample estimators yield largely congruent results. $N_{\mathrm{e}}$ and $N_{\mathrm{c}}$ ratios, however, are highly variable; both $N_{\mathrm{e}}$ and $N_{\mathrm{c}}$ strongly increase during the study period. The study, similar to the sparrow example, clearly shows the high value and importance of longterm monitoring and the potential for misleading results when only a single point in time is considered. In the final paper, Laikre et al. present a conservation study investigating the meta-population size of the Scandinavian wolf population based on empirically estimated demographic parameters. They find the currently realized $N_{\mathrm{e}}$ of the species in the region to be much below conservation targets. Conservation goals can only be reached by strongly increasing $N_{\mathrm{e}}$ and/or increasing immigration from larger Russian populations, as long as gene flow within the meta-population remains high.

The special issue covers a variety of topics from theoretical to applied questions related to $N_{\mathrm{e}}$, points to the direction in which research currently heads and highlights gaps that need to be filled in the future. It is quite clear that new technologies, especially in terms of data generation (next generation sequencing) and also analytical methods, will enrich not only the estimation of $N_{\mathrm{e}}$, but also the whole field of population and conservation genetics. We hope this special issue will spark discussion and encourage new research.

\section{CONFLICT OF INTEREST}

The authors declare no conflict of interest.

\section{ACKNOWLEDGEMENTS}

We thank all the authors for their contributions. Special thanks also go to the reviewers who helped to guarantee the high scientific standard of the articles.
We also want to express our gratitude to Mike Bruford and Mafalda Costa for the opportunity to edit this special issue and their help in composing it.

\author{
M Husemann ${ }^{1,2}$, FE Zachos ${ }^{3}$, RJ Paxton ${ }^{2}$ and JC Habel ${ }^{4}$ \\ ${ }^{1}$ Department of Entomology, Centrum für Naturkunde, \\ University of Hamburg, Hamburg, Germany; \\ ${ }^{2}$ General Zoology, Martin-Luther University Halle-Wittenberg, \\ Halle (Saale), Germany; \\ ${ }^{3}$ Natural History Museum Vienna, Vienna, Austria; \\ ${ }^{4}$ Terrestrial Ecology Research Group, Department of Ecology and \\ Ecosystem Management Universität München, Freising, Germany \\ E-mail: martinhusemann@yahoo.de
}

Braude S, Templeton AR (2009). Understanding the multiple meanings of 'inbreeding' and 'effective size' for genetic management of African rhinoceros populations. African J Ecol 47: 546-555

Coscia I, Chopelet J, Waples RS, Mann BQ, Mariani S (2016). Sex change and effective population size: implications for population genetic studies in marine fish. Heredity; 117: $251-258$

Cousseau L, Husemann M, Foppen R, Vangestel C, Lens L (2016). A longitudinal genetic survey identifies temporal shifts in the population structure of Dutch house sparrows. Heredity (Edinb) 117: 259-267.

Ferchaud A-L, Perrier C, April J, Hernandez C, Dionne M, Bernatchez L (2016). Making sense of the relationships between $\mathrm{Ne}, \mathrm{Nb}$ and $\mathrm{Nc}$ towards defining conservation thresholds in Atlantic salmon (Salmo salar) 117: 268-278.

Habel JC, Husemann M, Finger A, Danley PD, Zachos FE (2014). The relevance of time series in molecular ecology and conservation biology. Biol Rev Camb Philos Soc 89: 484-492.

Hollenbeck CM, Portnoy DS, Gold JR (2016). A method for detecting recent changes in contemporary effective population size from linkage disequilibrium at linked and unlinked loci. Heredity (Edinb) 117: 207-216.

Jones AT, Ovenden JR, Wang Y-G (2016). Improved confidence intervals for the linkage disequilibrium method for estimating effective population size Heredity 117: 217-223.

Laikre L, Olsson F, Jansson E, Hössjer O, Ryman N (2016). Metapopulation effective size and conservation genetic goals for the Fennoscandian wolf (Canis lupus) population Heredity 117: 279-289.

Mueller A-K, Chakarov N, Krüger O, Hoffman JI (2016). Long-term effective population size dynamics of an intensively monitored vertebrate population Heredity 117: 290-299.

Nunney L (2016). The effect of neighborhood size on effective population size in theory and in practice. Heredity 117: 224-232.

Wang J, Santiago E, Caballero A (2016). Prediction and estimation of effective population size Heredity 117: 193-206.

Waples RK, Larson WA, Waples RS (2016). Estimating contemporary effective population size in non-model species using linkage disequilibrium across thousands of loci. Heredity 117: 233-240.

Waples RS (2016). Life-history traits and effective population size in species with overlapping generations revisited: the importance of adult mortality. Heredity (Edinb) 117: 241-250. 